\title{
TU/e EN⿴HONE

\section{Dynamical modelling and control of snake-like motion in vertical plane for locomotion in unstructured environments}

\section{Citation for published version (APA):}

Koopaee, M. J., Pretty, C., Classens, K., \& Chen, X. Q. (2019). Dynamical modelling and control of snake-like motion in vertical plane for locomotion in unstructured environments. In 15th IEEE/ASME International Conference on Mechatronic and Embedded Systems and Applications (Vol. 9). [DETC2019-97227] (Proceedings of the ASME Design Engineering Technical Conference; Vol. 9). American Society of Mechanical Engineers. https://doi.org/10.1115/DETC2019-97227

DOI:

10.1115/DETC2019-97227

Document status and date:

Published: 25/11/2019

\section{Document Version:}

Accepted manuscript including changes made at the peer-review stage

\section{Please check the document version of this publication:}

- A submitted manuscript is the version of the article upon submission and before peer-review. There can be important differences between the submitted version and the official published version of record. People interested in the research are advised to contact the author for the final version of the publication, or visit the $\mathrm{DOI}$ to the publisher's website.

- The final author version and the galley proof are versions of the publication after peer review.

- The final published version features the final layout of the paper including the volume, issue and page numbers.

Link to publication

\section{General rights}

Copyright and moral rights for the publications made accessible in the public portal are retained by the authors and/or other copyright owners and it is a condition of accessing publications that users recognise and abide by the legal requirements associated with these rights.

- Users may download and print one copy of any publication from the public portal for the purpose of private study or research.

- You may not further distribute the material or use it for any profit-making activity or commercial gain

- You may freely distribute the URL identifying the publication in the public portal.

If the publication is distributed under the terms of Article 25fa of the Dutch Copyright Act, indicated by the "Taverne" license above, please follow below link for the End User Agreement:

www.tue.nl/taverne

Take down policy

If you believe that this document breaches copyright please contact us at:

openaccess@tue.nl

providing details and we will investigate your claim. 


\title{
Dynamical Modelling and Control of Snake-like Motion in Vertical Plane for Locomotion in Unstructured Environments
}

\author{
Mohammadali Javaheri Koopaee ${ }^{*}$, Christopher Pretty \\ Mechanical Engineering Department, \\ University of Canterbury, \\ Christchurch, New Zealand.
}

\author{
Koen Classens \\ Mechanical Engineering Department, \\ University of Eindhoven \\ AZ Eindhoven, Netherlands.
}

\author{
XiaoQi Chen ${ }^{\dagger}$ \\ Manufacturing Futures Research Institute \\ Swinburne University of Technology, Hawthorn, Australia.
}

\begin{abstract}
:
This paper introduces the equations of motion of modular $2 D$ snake robots in the vertical plane. In particular, the kinematics of pedal wave motion (undulation in vertical plane) of modular snake robots is presented and using the Euler-Lagrange method, the equations of motion of the robot are obtained. Moreover, using the well-known Spring-Damper contact model, external contact forces are taken into account and pedal wave locomotion on uneven terrain is modelled and simulated. Enabled by the dynamical model of the robot, an adaptive controller based on external force feedback in gait parameter space is proposed and implemented, resulting in the robot to successfully climbing over a stair-type obstacle without any prior knowledge about the environment.
\end{abstract}

Keywords: Bio inspired snake robots; equations of motion; pedal wave locomotion; adaptive locomotion; uneven terrain.

\section{INTRODUCTION}

There are many life forms with incredibly effective locomotion mechanisms, sensing and computation capabilities, which are invaluable sources of inspiration for researchers. One of these bio-inspired designs are snake-like robots [1], which their small body cross section, intrinsic stability, maneuverability and hyper redundancy have made them ideal for locomotion in challenging environments, such as pipes [2], tankers [3] and collapsed buildings [4].

Generally speaking, biological snakes can perform a number of locomotion patterns based on environmental conditions to effectively traverse in challenging environments. Among such motion patterns, lateral undulation [5] and its counterpart in vertical plane (rectilinear [6], or pedal wave motion [7]) are of special interest due to their intrinsic ability to move in unstructured environments with different features.

Lateral undulation, which is the most common locomotion pattern among biological snakes has received considerable attention since studies on snake robots started. Most of these studies consider installing wheels to mimic the anisotropic friction properties of real snakes that makes this gait effective [8], however, such wheeled snake robots suffer from the same limitations of wheeled mobile robots, hence, they are not suitable for locomotion in unstructured environments. Moreover, although it has been shown that anisotropic friction property of snake belly scales and existence of push points in the environment are the major factors for generating such forward motion [9], development of snake-like skin, such as [10] has not yet generated practical results.

To overcome the aforementioned issues, some recent works such as [11], have focused on designing a robot capable of pushing against the irregularities of the terrain to move forward. Although such obstacle aided locomotion patterns have been shown to be very effective [12], existence of sufficient number of pegs for the robot to push against is essential to generate enough propulsive forces. Hence, such locomotion mechanisms are effective only for locomotion in confined spaces, such as pipes where the robot can always push against the walls to move forward.

On the other hand, pedal wave motion of snake robots, which is similar to caterpillar motion [13], is an effective locomotion pattern very similar to lateral undulation but performed in vertical plane. In this type of motion, the robot lifts some of its links and pushes against the ground to move

\footnotetext{
*Corresponding Author, Mohammadali.javaherikoopaee@pg.canterbury.ac.nz

$\dagger$ “Previously affiliated with Mechanical Engineering Department, University of Canterbury, Christchurch, New Zealand”
} 
forward, which making it very effective for locomotion on uneven terrain, where lateral undulation is no longer a useful locomotion pattern [7]. Using such a locomotion pattern, one can take advantage of small cross section of snake body to move in confined spaces and because the robot pushes against the ground to move forward, existence of sideways push points no longer have an effect on generation of such movement.

Although pedal wave locomotion patterns can be used in challenging environments, dynamical modelling of such locomotion mechanisms, necessary for in depth investigation of the motion is challenging due to the contact between the robot links and the environment. Some recent works such as [6] have proposed a modelling framework based on series of connected mass and springs to model such motion, which is only suitable for biological snakes modelling. More recently, in [14] based on the well-known Newton principle and in [15] using the Euler-Lagrange method the dynamical equation of pedal wave motion is obtained. However, in both of these works it is assumed that the number of contact points remains constant during the motion, and the normal forces are obtained based on the force and moment balance. Hence, such models are not suitable for modelling a snake robot with multiple contact points in unstructured environments, where the robot might be in contact with multiple contact point in different planes.

In addition to the lack of a generalized modelling framework, the number of works which have focused on adaptive pedal wave motion are also limited. In [7] a shapebased control scheme has been proposed for pedal wave locomotion of snakes. However the proposed method requires prior information about the environment. In [16], a control mechanism based on torque control is proposed for a snake-like robot, However, the controller requires information from pressure sensor on the surface of the links. Consequently, a dynamical model of the robot, taking into account external forces from the environment could be very beneficial to test different adaptive controllers for pedal wave locomotion of snake robots.

In this paper, dynamical model of modular 2D snake robots performing pedal wave locomotion in vertical plane is presented. The kinematics of the robot have been obtained in matrix from and using the Euler-Lagrange method, the equation of motion of the robot has been derived. Using the well-known spring-damper contact model the effect of external forces on the robot are taken into account, which unlike existing models such as [15] can be used to model the robot in contact with the environments at multiple points in different planes. Additionally, it has been shown that such a dynamical model is flexible enough to simulate both lateral undulation inside a pipe and pedal wave motion. Moreover, using the obtained dynamical model, the robot is simulated in an environment with a stair type obstacle and an adaptive controller in gait parameters space is designed and effectiveness of such controller for climbing over the obstacle without prior knowledge about its location is shown.

The organization of this paper is as follows. In Section 1, the problem definition is discussed and pedal wave motion of modular snake robot is introduced. In Section 2, the kinematics of the planar snake robot is presented and in Section 3, the generalized equations of motion of the robot are obtained. Finally, in Section 4, modelling of the robot in unstructured environment is discussed and an adaptive controller based on external force feedback has been designed and the effectiveness of such controller is supported by simulation results.

\section{BACKGROUND}

Today's world of robotic is completely dominated by wheeled robots, however, this popularity stems from simplicity in modelling, control and manufacture of such robots, and not their capabilities to overcome locomotion challenges. On the other hand snake-like robots have proved to be very effective in unstructured environments, where for wheeled robotic systems is impossible to operate.

Among snake-like locomotion patterns, pedal wave motion has number of advantages over other gaits in unstructured environments. This type of locomotion, which is similar to extended caterpillar locomotion with multiple contact points, is exhibited mostly in heavy snakes. Former studies on this type of locomotion suggested that, in pedal wave (rectilinear) locomotion snake ribs act as legs, similar to walking. However the snake does not "travel on its ribs", [17] or in the other words the snake ribs are not the main means of locomotion.

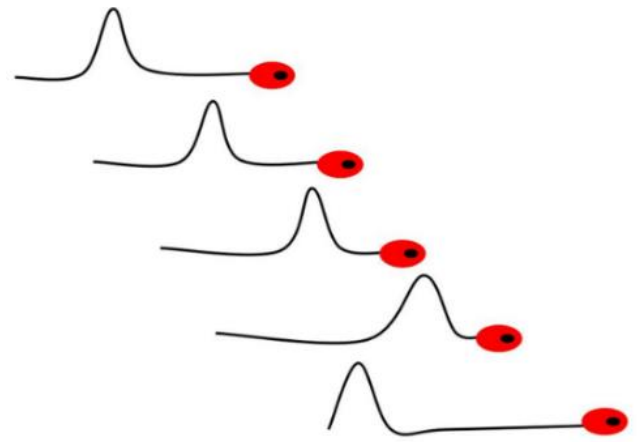

FIGURE 1: PEDAL WAVE (RECTILINEAR) LOCOMOTION

In rectilinear motion, similar to earthworms, the snake travels in a straight line, and unlike the other type of locomotion, sideways interaction with the environment is not essential. Instead, contraction and relaxation waves pass over the ventral muscles along with lifting body parts are the main cause of locomotion [6]. FIGURE 1 displays the fundamental features of such a locomotion pattern.

To mimic such motion, different designs, such as [16], are proposed. However the most common snake-like mechanism, capable of performing pedal wave motion is shown in FIGURE 2 [18], where a snake robot consists of six links and five servos rotating about a common axes has been developed. This robot is capable of generating pedal wave motion by controlling the joints angles based on based on the following gait pattern, inspired by the body shape of biological snakes

$$
\varphi_{j}^{d}(t)=A_{p} \sin (\omega t+\eta(j-1))
$$

where $\varphi_{j}^{d} ; j=1,2, \ldots, N-1$ are the desired relative joint angles, $\omega$ is the temporal frequency, $\eta$ is the spatial frequency and $A_{p}$ is the amplitude of the sinoisdal wave. FIGURE 2 shows a snake robot developed by the authors whit six links performing this type of motion with $A_{p}=\frac{\pi}{6} \mathrm{rad}, \omega=$ $\pi \mathrm{rad} / \mathrm{sec}$ and $\eta=\frac{-2 \pi}{5} \mathrm{rad}$. 
One of the most important features of pedal wave motion is that the snake can lift part of its body part and push against the ground to move forward, making it very suitable for locomotion on uneven terrain. However, this also makes modelling of such motion challenging, compared to lateral undulation, in which the robot body is constantly in contact with ground. Consequently, any dynamical model of pedal wave motion should deal with contact forces in an efficient manner to obtain a suitable simulation model to be used for investigating such a complex motion and possibly used for designing an adaptive motion to move in unstructured environment.

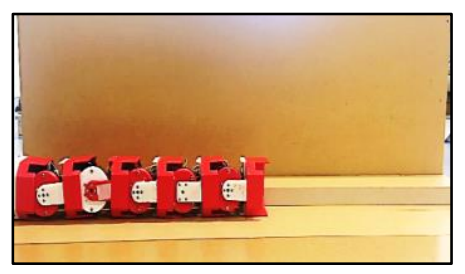

(a) $t=0$

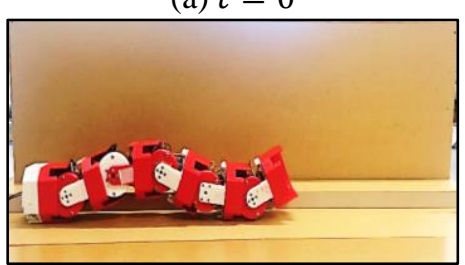

(b) $t=2 \mathrm{~s}$

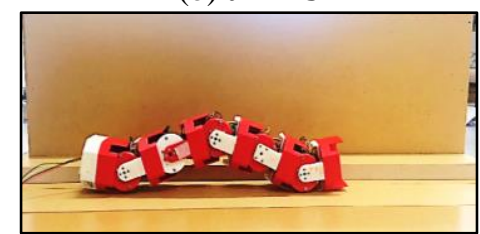

(c) $t=4 \mathrm{~s}$

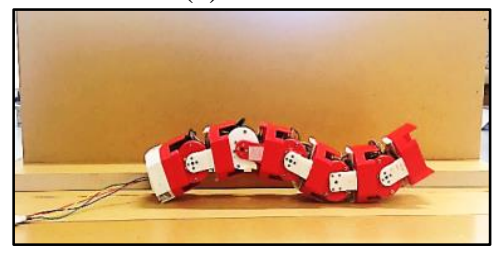

(d) $t=6 \mathrm{~s}$

FIGURE 2: THE SNAKE ROBOT DEVELOPED BY THE AUTHORS PERFORMING PEDAL WAVE MOTION

In the next section, we will mainly focus on obtaining the kinematics of such motion in XZ plane in a matrix form suitable for the Euler-Lagrange method.

\section{KINEMATIC OF 2D SNAKE ROBOTS}

Consider the body shape of a 2D snake robot, as shown in FIGURE 3. For this model $\theta_{i}, i=1,2, \ldots, N$ is the absolute link angle of $i^{\text {th }}$ link, $2 l$ is the length of each identical link $\varphi_{j}, j=1,2, \ldots, N-1$ is the relative angle between the links, $\left[x_{i}, z_{i}\right]$ is the position of center of mass of $i^{t h}$ link in the global coordinate frame and $\left[p_{x}, p_{z}\right]$ is the position of center of mass of the robot.

Considering the planar body shape of the robot, the position of center of mass of each link can be obtained as the function of absolute link angles as follows:

$$
\begin{aligned}
& x_{i}=x_{0}+2 l \sum_{j=1}^{i-1} \cos \left(\theta_{j}\right)+l \cos \left(\theta_{i}\right) \\
& z_{i}=z_{0}+2 l \sum_{j=1}^{i-1} \sin \left(\theta_{j}\right)+l \sin \left(\theta_{i}\right),
\end{aligned}
$$

Noting that $\left[p_{x}, p_{z}\right]=1 / N\left[\sum_{i=1}^{N} x_{i}, \sum_{i=1}^{N} z_{i}\right]$ and replacing $x_{i}$ and $z_{i}$ from (2), the following relations can be obtained:

$$
\begin{aligned}
& x_{i}= p_{x}+2 l \sum_{j=1}^{i-1} \cos \left(\theta_{j}\right)+l \cos \left(\theta_{i}\right) \\
&-1 / N \sum_{n=1}^{N}\left(2 l \sum_{j=1}^{n-1} \cos \left(\theta_{j}\right)+l \cos \left(\theta_{n}\right)\right) \\
& z_{i}=p_{z}+2 l \sum_{j=1}^{i-1} \sin \left(\theta_{j}\right)+l \sin \left(\theta_{i}\right) \\
&-1 / N \sum_{n=1}^{N}\left(2 l \sum_{j=1}^{n-1} \sin \left(\theta_{j}\right)+l \sin \left(\theta_{n}\right)\right)
\end{aligned}
$$

Consequently, considering (3), the time derivative of $x_{i}$ and $z_{i}$ can easily be obtained to be as follows:

$$
\begin{gathered}
\dot{x}_{i}=\dot{p}_{x}-2 l \sum_{j=1}^{i-1} \sin \left(\theta_{j}\right) \dot{\theta}_{j}-l \sin \left(\theta_{i}\right) \dot{\theta}_{i} \\
+1 / N \sum_{n=1}^{N}\left(2 l \sum_{j=1}^{n-1} \sin \left(\theta_{j}\right) \dot{\theta}_{j}+l \sin \left(\theta_{n}\right) \dot{\theta}_{n}\right) \\
\dot{z}_{i}=\dot{p}_{z}+2 l \sum_{j=1}^{i-1} \cos \left(\theta_{j}\right) \dot{\theta}_{j}+l \cos \left(\theta_{i}\right) \dot{\theta}_{i} \\
-1 / N \sum_{n=1}^{N}\left(2 l \sum_{j=1}^{n-1} \cos \left(\theta_{j}\right) \dot{\theta}_{j}+l \cos \left(\theta_{n}\right) \dot{\theta}_{n}\right)
\end{gathered}
$$

which together with (3) describe the position and velocity of center of mass of each link as functions of absolute link angles expressed in the global coordinate frame. However, to make the derivation and implementation of Euler-Lagrange equations to be presented in the next section straightforward, it is more convenient to represent these equations in matrix form. Hence, we can rewrite (2) as follows:

$$
\begin{aligned}
& \boldsymbol{X}=\boldsymbol{V} p_{x}-\frac{1}{N} \boldsymbol{V} \boldsymbol{V}^{T} \mathcal{H} \boldsymbol{C} \boldsymbol{\theta}+\mathcal{H} \boldsymbol{C} \boldsymbol{\theta} \\
& \boldsymbol{Z}=\boldsymbol{V} p_{z}-\frac{1}{N} \boldsymbol{V} \boldsymbol{V}^{T} \mathcal{H} \boldsymbol{S} \boldsymbol{\theta}+\mathcal{H} \boldsymbol{S} \boldsymbol{\theta}
\end{aligned}
$$

where $\boldsymbol{X}=\left[x_{1}, x_{2}, \ldots, x_{N}\right]^{T}, \quad \boldsymbol{Z}=\left[z_{1}, z_{2}, \ldots, z_{N}\right]^{T}, \quad \boldsymbol{V}_{N \times 1}=$ $\left[\begin{array}{llll}1 & 1 & \ldots & 1\end{array}\right]^{T}, \mathcal{H}$ is an $N$ by $N$ constant matrix defined as follows: 

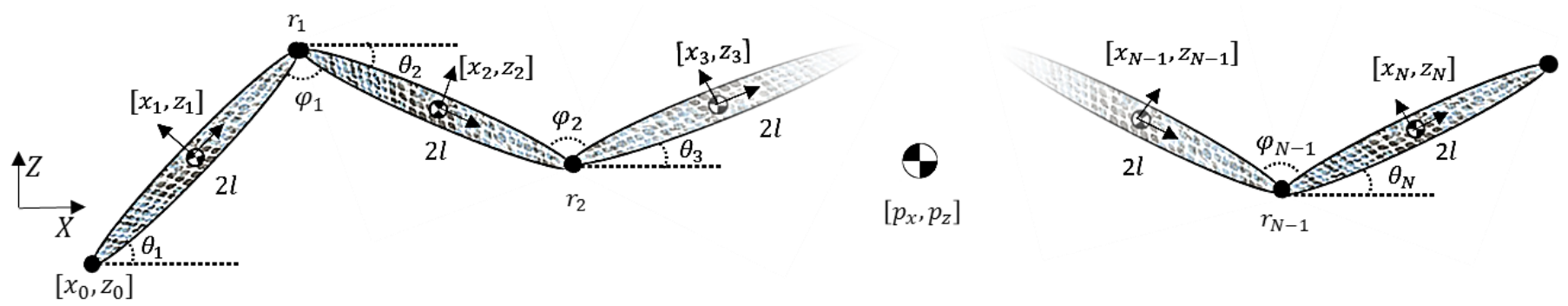

FIGURE 3: BODY SHAPE OF THE ROBOT PERFORMING PEDAL WAVE MOTION

$$
\mathcal{H}^{l}=\left[\begin{array}{ccccc}
l & 0 & 0 & \ldots & 0 \\
2 l & l & 0 & \ldots & 0 \\
2 l & 2 l & l & \ldots & 0 \\
\vdots & \vdots & \vdots & \vdots & \vdots \\
2 l & 2 l & 2 l & \ldots & l
\end{array}\right]_{N \times N}
$$

undulation locomotion in horizontal plane XY by only replacing $\mathrm{Z}$ axis with $\mathrm{Y}$ axis. For example, equation (2) can easily be modified as follows to describe the position of each robot link when performing lateral undulation in XY plane.

$$
\begin{aligned}
& x_{i}=x_{0}+2 l \sum_{j=1}^{i-1} \cos \left(\theta_{j}\right)+l \cos \left(\theta_{i}\right) \\
& y_{i}=y_{0}+2 l \sum_{j=1}^{i-1} \sin \left(\theta_{j}\right)+l \sin \left(\theta_{i}\right),
\end{aligned}
$$

Using this notation, the gradient of (5) with respect to time can easily be obtained to be as follows:

$$
\begin{aligned}
& \dot{\boldsymbol{X}}=\boldsymbol{V} \dot{p}_{x}+\frac{1}{N} \boldsymbol{V} \boldsymbol{V}^{T} \mathcal{H} \mathcal{S}_{\theta} \dot{\boldsymbol{\theta}}-\mathcal{H} \mathcal{S}_{\theta} \dot{\boldsymbol{\theta}} \\
& \dot{\boldsymbol{Z}}=\boldsymbol{V} \dot{p}_{z}-\frac{1}{N} \boldsymbol{V} \boldsymbol{V}^{T} \mathcal{H} \mathcal{C}_{\theta} \dot{\boldsymbol{\theta}}+\mathcal{H} \mathcal{C}_{\theta} \dot{\boldsymbol{\theta}}
\end{aligned}
$$

where $\dot{\boldsymbol{\theta}}=\left[\dot{\theta}_{1}, \dot{\theta}_{2}, \ldots, \dot{\theta}_{N}\right]$ and

$$
\begin{aligned}
& \mathcal{S}_{\theta}=\operatorname{diag}\left(\sin \left(\theta_{1}\right), \sin \left(\theta_{2}\right), \ldots, \sin \left(\theta_{N}\right)\right), \\
& \mathcal{C}_{\theta}=\operatorname{diag}\left(\cos \left(\theta_{1}\right), \cos \left(\theta_{2}\right), \ldots, \cos \left(\theta_{N}\right)\right) .
\end{aligned}
$$

Finally, noting that $\theta_{i}=\sum_{n=i}^{N-1} \varphi_{n}+\theta_{N}$, the absolute joint angle vector $\boldsymbol{\theta}$ can be written as follows:

$$
\boldsymbol{\theta}=\mathcal{F} \boldsymbol{\varphi}+\boldsymbol{V} \theta_{N}
$$

where $\boldsymbol{\varphi}=\left[\begin{array}{llll}\varphi_{1} & \varphi_{2} & \ldots & \varphi_{N-1}\end{array}\right]^{T}$ and $\mathcal{F}$ is an $N$ by $N-1$ constant matrix defined below:

$$
\mathcal{F}=\left[\begin{array}{ccccc}
1 & 1 & 1 & \ldots & 1 \\
0 & 1 & 1 & \ldots & 1 \\
0 & 0 & 1 & \ldots & 1 \\
\vdots & \vdots & \vdots & \vdots & \vdots \\
0 & 0 & 0 & \ldots & 0
\end{array}\right]_{N \times N-1}
$$

It should be noted that the presented procedure so far made it possible to obtain the position and velocity of the center of mass of each link as a function of joint angles $\varphi_{i}$, the absolute angle of the head module and the position of center of mass of the robot in matrix form. Hence to implement such equations no symbolic computation is necessary, making the implementation of equation of motion to be presented in the next section simpler.

It should also be mentioned that although the kinematic relations obtained for pedal wave in the vertical plane XZ, the same equations can describe the kinematic relations of lateral

where $\left[x_{i}, y_{i}\right]$ is the position of center of mass of $i^{t h}$ link in the global coordinate frame OXY and $\left[p_{x}, p_{y}\right]$ is the position of center of mass of the robot.

\section{DYNAMICAL EQUATION}

Choosing the generalized coordinates, as $\boldsymbol{q}=$ $\left[\varphi_{1}, \varphi_{2}, \ldots, \varphi_{n-1}, \theta_{N}, p_{x}, p_{y}\right]$, the Euler-Lagrange equations for describing the dynamics of snake robot can immediately be constructed as follows:

$$
\frac{d}{d t}\left(\frac{\partial T}{\partial \dot{\boldsymbol{q}}}\right)-\frac{\partial T}{\partial \boldsymbol{q}}+\frac{\partial \mathcal{V}}{\partial \boldsymbol{q}}=\boldsymbol{Q}^{\tau}+\boldsymbol{Q}^{f}
$$

where $\boldsymbol{Q}^{\tau}$ and $\boldsymbol{Q}^{f}$ are the vectors of non-conservative generalized torques and forces, i.e. control inputs, friction and other environmental forces, $T$ is the sum of kinetic energy of the links and $V$ is the potential energy of the system due to the gravity.

\section{Kinetic Energy}

To obtain the expression for kinetic energy of the links it is necessary to obtain the velocity of each link as a function of generalized coordinates $\boldsymbol{q}$. This can be done by rewriting (6) and (7) as follows:

$$
\begin{aligned}
& \dot{\boldsymbol{X}}= {\left[\begin{array}{lll}
0_{N \times N-1} & \boldsymbol{V} & \mathbf{0}_{\boldsymbol{N} \times \mathbf{1}}
\end{array}\right] \dot{\boldsymbol{q}} } \\
&+\left\{\begin{array}{lll}
-\mathcal{H}+\frac{\mathbf{1}}{N} \boldsymbol{V} \boldsymbol{V}^{\boldsymbol{T}} \mathcal{H}
\end{array}\right\} \mathcal{S}_{\theta}\left[\begin{array}{lll}
\mathcal{F} & \boldsymbol{V} & 0_{N \times 2}
\end{array}\right] \dot{\boldsymbol{q}} \\
& \dot{\boldsymbol{Z}}=\left[\begin{array}{lll}
0_{N \times N-1} & \boldsymbol{0}_{\boldsymbol{N} \times \mathbf{1}} & \boldsymbol{V}
\end{array}\right] \dot{\boldsymbol{q}} \\
&+\left\{\begin{array}{lll}
\mathcal{H}-\frac{1}{N} \boldsymbol{V} \boldsymbol{V}^{\boldsymbol{T}} \mathcal{H}
\end{array}\right\} \mathcal{C}_{\theta}\left[\begin{array}{lll}
\mathcal{F} & \boldsymbol{V} & 0_{N \times 2}
\end{array}\right] \dot{\boldsymbol{q}} \\
& \dot{\boldsymbol{\theta}}=\left[\begin{array}{lll}
F & \boldsymbol{V} & 0_{N \times 2}
\end{array}\right] \dot{\boldsymbol{q}} .
\end{aligned}
$$

Defining

$$
\begin{aligned}
& B_{1_{N \times(N+2)}}=\left[\begin{array}{lll}
0_{N \times N-1} & \boldsymbol{V} & \mathbf{0}_{N \times 1}
\end{array}\right] \\
& B_{2_{N \times(N+2)}}=\left[\begin{array}{lll}
0_{N \times N-1} & \mathbf{0}_{N \times 1} & \boldsymbol{V}
\end{array}\right]
\end{aligned}
$$


and

$$
\begin{gathered}
A_{N \times N}=-\mathcal{H}+\frac{1}{N} \boldsymbol{V} \boldsymbol{V}^{T} \mathcal{H} \\
C_{N \times(N+2)}=\left[\begin{array}{lll}
\mathcal{F} & \boldsymbol{V} & 0_{N \times 2}
\end{array}\right],
\end{gathered}
$$

and finally considering (10), the expression for $\dot{\boldsymbol{X}}, \dot{\boldsymbol{Z}}$ and $\dot{\boldsymbol{\theta}}$ can be obtained as follows:

$$
\begin{gathered}
\dot{\boldsymbol{X}}=\left(B_{1}+A \mathcal{S}_{\theta} C\right) \dot{\boldsymbol{q}} \\
\dot{\boldsymbol{Z}}=\left(B_{2}-A \mathcal{C}_{\theta} C\right) \dot{\boldsymbol{q}} \\
\dot{\boldsymbol{\theta}}=C \dot{\boldsymbol{q}}
\end{gathered}
$$

and consequently the expression for the kinetic energy of the system can be constructed as below:

$$
T=\frac{m}{2} \dot{\boldsymbol{X}}^{T} \dot{\boldsymbol{X}}+\frac{m}{2} \dot{\boldsymbol{Z}}^{T} \dot{\boldsymbol{Z}}+\frac{J}{2} \dot{\boldsymbol{\theta}}^{T} \dot{\boldsymbol{\theta}}
$$

where $m$ and $J$ are the mass and moment of inertial of each link identical respectively. It should be noted that to fully obtain the equation of motion of the robot, derivative of $T$ with respect to $\boldsymbol{q}$ and $\dot{\boldsymbol{q}}$ needs to obtained, which considering the matrix form presented will be straight forward, hence neglected here for the sake of brevity.

\section{Potential Energy}

Unlike lateral undulation in confined spaces, in which the robot is not subject to any gravitational force, in pedal wave motion the robot lift its body part from the ground. Thus considering the body shape of the robot in $\mathrm{XZ}$ plane, the expression for potential energy of the system can be obtained as:

$$
\mathcal{V}=m g \boldsymbol{V}^{\boldsymbol{T}} \boldsymbol{Z}=m g \boldsymbol{V}^{\boldsymbol{T}}\left(\boldsymbol{V} z_{0}+\mathcal{H} \boldsymbol{S} \boldsymbol{\theta}\right),
$$

Substituting $z_{0}$ from the expression of center of mass of the robot, (13) can be written as follows:

$$
\mathcal{V}=m g \boldsymbol{V}^{\boldsymbol{T}} \boldsymbol{Z}=m g \boldsymbol{V}^{\boldsymbol{T}}\left(B_{2} \boldsymbol{q}-A \boldsymbol{S} \boldsymbol{\theta}\right),
$$

Finally, the derivative of $\mathcal{V}$ with respect to $\boldsymbol{q}$ can be obtained to be:

$$
\frac{\partial \mathcal{V}}{\partial \boldsymbol{q}}=\mathcal{G}=m g \boldsymbol{V}^{T}\left(B_{2}-A \mathcal{C}_{\theta} C\right)
$$

which will be directly incorporated into the equation of motion.

\section{Non Conservative Forces}

To obtain the effect of non-conservative forces $f_{i}^{x}, f_{i}^{z}$ on $i^{\text {th }}$ link of the robot expressed in the global coordinate frame, it is enough to consider the point of effect of such forces. Assuming that these forces will be exerted on the center of mass of each link, $Q^{f}$ can be obtained as follows:

$$
\boldsymbol{Q}^{f}=\sum_{i=1}^{N}\left[\begin{array}{ll}
\frac{\partial \dot{x}_{i}^{l}}{\partial \dot{\boldsymbol{q}}} & \frac{\partial \dot{z}_{i}^{l}}{\partial \dot{\boldsymbol{q}}}
\end{array}\right]^{T}\left[\begin{array}{c}
f_{i}^{x} \\
f_{i}^{z}
\end{array}\right]
$$

which can be written in matrix for as:

$$
\boldsymbol{Q}^{f}=\left[\begin{array}{ll}
\frac{\partial \mathrm{X}^{\mathrm{T}}}{\partial \boldsymbol{q}} & \frac{\partial \mathrm{Z}^{\mathrm{T}}}{\partial \boldsymbol{q}}
\end{array}\right]\left[\begin{array}{l}
\boldsymbol{F}^{x} \\
\boldsymbol{F}^{z}
\end{array}\right]
$$

where $\frac{\partial \mathrm{x}}{\partial \boldsymbol{q}}=\left(B_{1}+A \mathcal{S}_{\theta} C\right), \quad \frac{\partial \mathrm{Z}}{\partial \boldsymbol{q}}=\left(B_{2}-A \mathcal{C}_{\theta} C\right)$ and $\boldsymbol{F}^{x}=$ $\left[\begin{array}{llll}f_{x 1} & f_{x 2} & \ldots & f_{x N}\end{array}\right]^{T}, \boldsymbol{F}^{Z}=\left[\begin{array}{llll}f_{z 1} & f_{z 2} & \ldots & f_{z N}\end{array}\right]^{T}$ are the vectors of all non-conservative forces along the global $X$ and $Z$ direction respectively.

The above procedure can be used to take into account any non-conservative forces such as friction and/or contact forces, necessary to model both pedal wave motion and lateral undulation in confined spaces. For example, FIGURE 4 shows a general case where a single link of the robot is in contact with an obstacle and $f_{k}^{N}$ and $f_{k}^{T}$ are the normal and tangential forces exerted on the robot at the point $p_{k}$ by the environment.

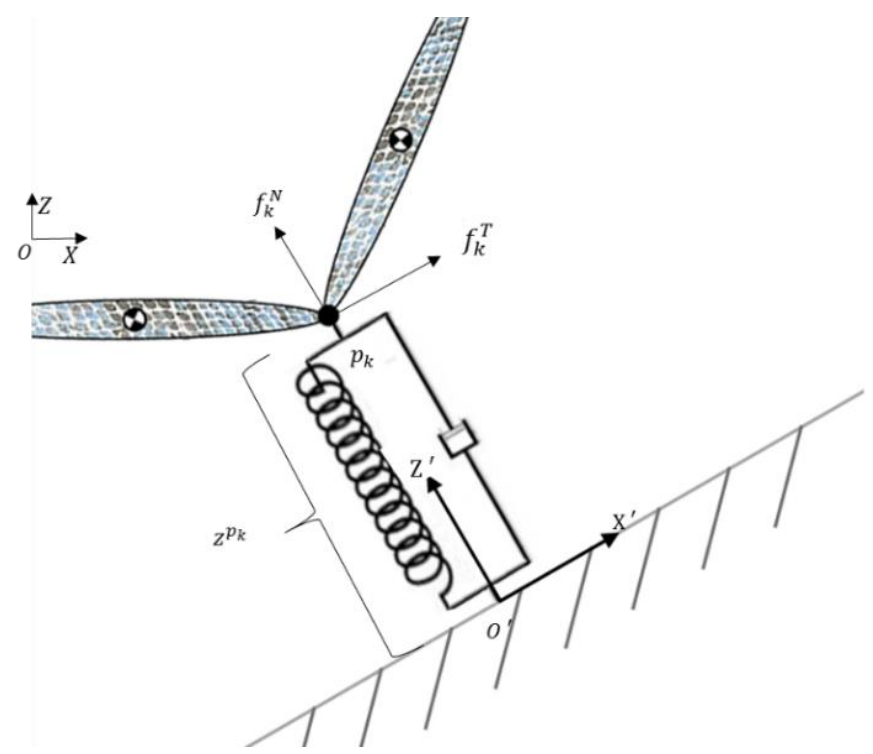

FIGURE 4: SPRING-DAMPER CONTACT MODEL

Assuming that $p_{k}$ is in contact with the obstacle, i.e. $z^{p_{k}} \leq$ 0 , and considering a spring-damper contact model, $f_{k}^{N}$ can be calculated as follows:

$$
f_{k}^{N}=\operatorname{Max}\left(k\left(z^{p_{k}}\right)-d \dot{z}^{p_{k}}, 0\right)
$$

where $z^{p_{k}}$ is the coordinate of the point of contact $p_{k}$ along the $Z^{\prime}$ direction of the frame $O X^{\prime} Z^{\prime}, k$ is the spring and $d$ is the damping constant of the environment. Once $f_{k}^{N}$ is obtained, it is straightforward to calculate $f_{k}^{T}$ as follows:

$$
f_{k}^{T}=-f_{k}^{N} \mu_{C} \operatorname{sign}\left(\dot{x}^{p_{k}}\right)
$$

where $\dot{x}^{p_{k}}$ is the velocity of the point $p_{k}$ along the direction tangent to the surface and $\mu_{C}$ is the friction coefficient.

Having derived $f_{k}^{N}$ and $f_{k}^{T}$, it will be straight forward to incorporate these forces into the equations of motion. However as shown in the general case of FIGURE 4 , forces $f_{k}^{N}$ and $f_{k}^{T}$ are expressed in the stationary coordinate frame $O X^{\prime} Z^{\prime}$, which is not necessary aligned with the global coordinate frame, thus these forces should be expressed in the global coordinate frame 
to construct $\boldsymbol{F}_{x}$ and $\boldsymbol{F}_{z}$ and then use equation (17) to incorporate these forces into the dynamical model.

Another important consideration is that unlike friction forces, which are assumed to be exerted on the center of mass of each link, to obtain a more realistic simulation model of the robot, the effect of external forces on the robot joints, tip of the head and the tail should also be considered. That is the reason why in FIGURE 4 the external forces are assumed to be exerted at the joints as a general case. However, for this case instead of using (17) one should obtain the derivative of the position of the new contact points with respect to $\mathbf{q}$, which is straight forward and neglected here for the sake of brevity.

\section{Equations of Motion}

Considering the expression obtained for the kinetic and potential energies of the snake robot, it is now possible to construct the equation of motion for pedal wave motion as follows:

$$
M(q) \ddot{q}+\boldsymbol{C}(q, \dot{q})+\boldsymbol{G}=\boldsymbol{Q}^{\tau}+\boldsymbol{Q}_{1}^{f}+\boldsymbol{Q}_{2}^{f}
$$

where $M(q)_{(N+2) \times(N+2)}$ is the positive definite link inertia matrix, $\boldsymbol{C}(q, \dot{q})_{(N+2)}$ is the vector of Coriolis and centrifugal terms as presented in Appendix A, $\mathcal{G}$ is a column vector of gravitational forces, $\boldsymbol{Q}^{\tau}$ is the vector of control inputs, $\boldsymbol{Q}_{1}^{f}$ is the vector of friction forces and $\boldsymbol{Q}_{2}^{f}$ is the vector of other external forces due to contact with environment.

Similar equations can be used to describe lateral undulation, which is essentially the same type of motion in horizontal plane and thus not subject to gravity. Hence, the equation of motion of the snake robot performing lateral undulation can be obtained as follows:

$$
M(q) \ddot{q}+\boldsymbol{C}(q, \dot{q})=\boldsymbol{Q}^{\tau}+\boldsymbol{Q}_{1}^{f}+\boldsymbol{Q}_{2}^{f}
$$

where the definition of $M(q)_{(N+2) \times(N+2)}, \boldsymbol{C}(q, \dot{q})_{(N+2)}, \boldsymbol{Q}^{\tau}$, $\boldsymbol{Q}_{1}^{f}, \boldsymbol{Q}_{2}^{f}$ will remain the same as (20).

Both equations (20) and (21) have the same structure and the only difference is the gravity term presented to the equations of motion of pedal wave motion. Moreover, both (20) and (21) can be seen as two sets of equations. The first $N-1$ equations are the actuated dynamics of the system containing relative joint angles and the input torque and the last three equations are the dynamics of the head angle and position of the center of mass in addition frictional and external forces.

\section{Motion Generation}

To demonstrate the effectiveness of the proposed model in (20) generating pedal wave motion, the gait patterns (1) with $A_{p}=1.1 \mathrm{rad}, \omega=\frac{2 \pi}{5} \mathrm{rad} / \mathrm{sec}$ and $\eta=\frac{-\pi}{2} \mathrm{rad}$ has been implemented on both dynamical model (20) and (21). For this purpose, a snake robot with six identical links each with the mass of $0.1 \mathrm{~kg}$ and length $0.1 \mathrm{~m}$ has been simulated via MatlabR2017b, and the results demonstrating pedal wave motion are shown in FIGURE 5.
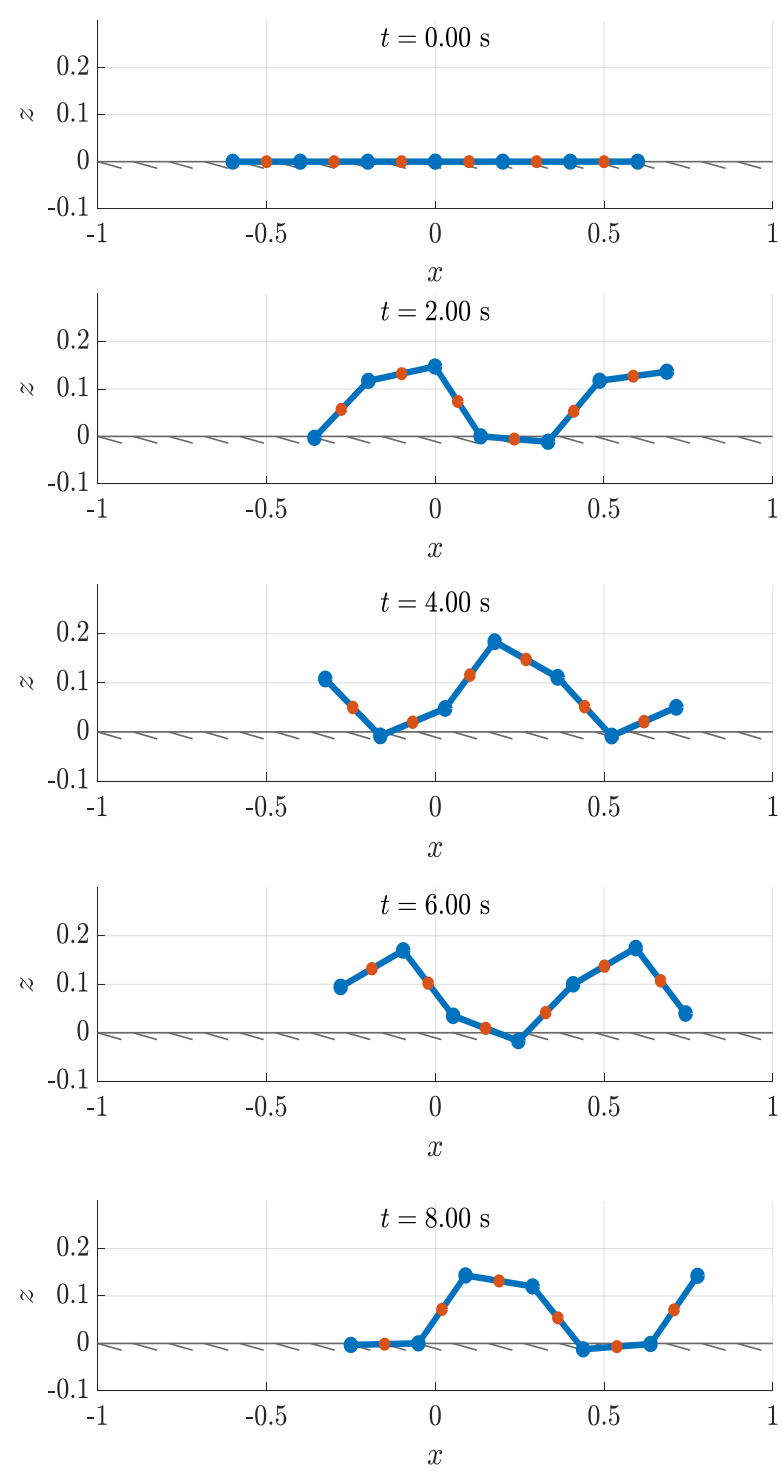

FIGURE 5: SIMULATED PEDAL WAVE MOTION

As shown in FIGURE 5, the proposed dynamical model (20) has been successfully used to simulate pedal wave motion when the spring and damping constant are chosen to be 350 and 25 respectively with the step time chosen to be 0.002 s. It should be noted that although at each time step all the possible contact points needs to be checked to calculate the external forces, it only took $4.21 \mathrm{sec}$ to simulate the motion, shown in FIGURE 5 .

Similar model can be used for simulation of lateral undulation inside a pipe based on (21). FIGURE 6 shows the snake robot with the same simulation parameters moving inside a pipe with the diameter of $20 \mathrm{~cm}$. Although based on the proposed dynamical model anisotropic friction force can easily be incorporated, to model the motion of the robot inside the pipe, in FIGURE 6 the only propulsive force comes from the interaction between the robot and pipe walls.

In the next section, we will focus on simulating the pedal wave motion in unstructured environment and use the proposed spring damper contact model to investigate adaptive pedal wave motion. 


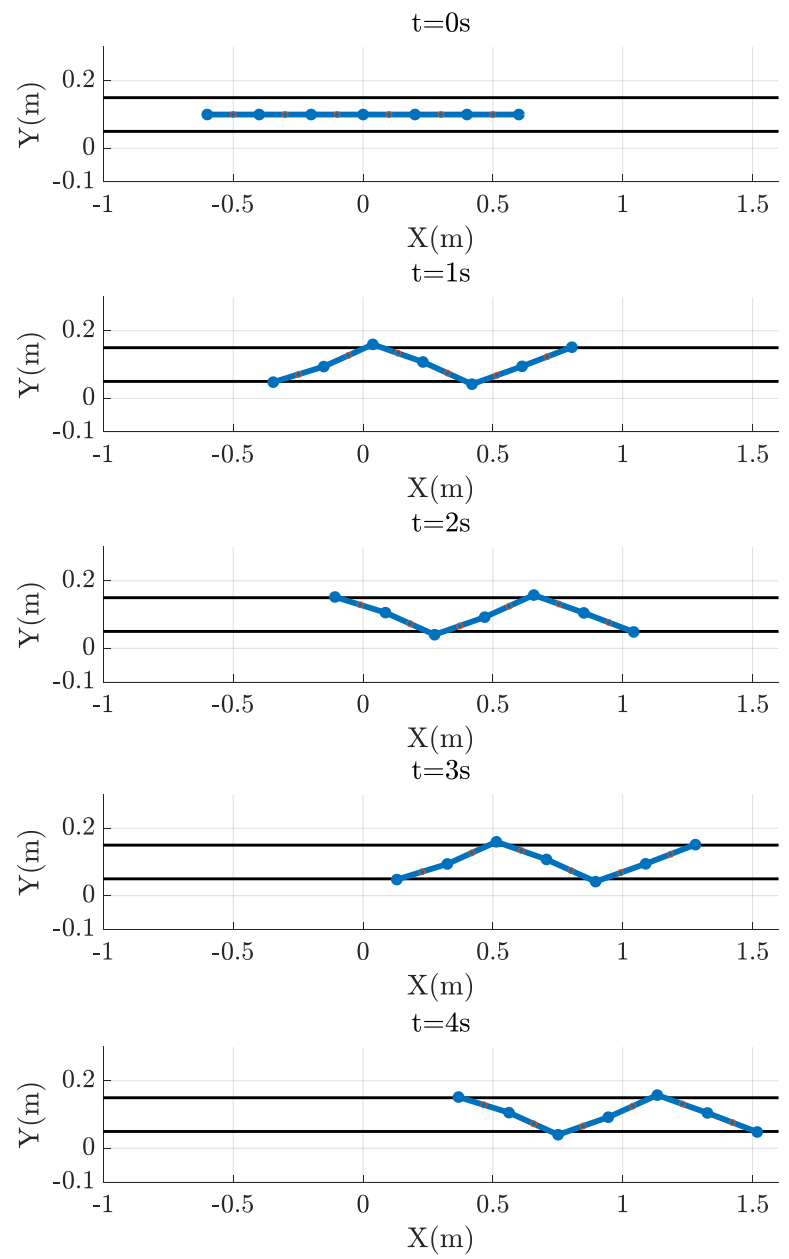

FIGURE 6: SIMULATED OF LATERAL UNDULATION INSIDE A PIPE

\section{ADAPTIVE PEDAL WAVE MOTION}

The dynamical model of the pedal wave motion of snake robots (20) can be extended to simulate locomotion of the robot in unstructured environments. This enables us to investigate the effect of adaptive control strategies for locomotion on uneven terrain. For this purpose, motivated by the controller proposed in [19] for lateral undulation, we propose a similar adaptive control strategy based on a feedback signal $\boldsymbol{Q}_{2}^{f}=$ $\left[Q_{2}^{f}, Q_{2}^{f}, \ldots, Q_{2 N}^{f}\right]^{T}$ (i.e. the vector of contact forces) for pedal wave motion based on the following extended gait pattern:

$$
\varphi_{j}^{d}\left(t, \eta_{j}\right)=A_{p} \sin \left(\omega t+\eta_{j}(j-1)\right)
$$

where $\eta_{j}$ is the spatial frequency of the sinoisdal command of $j^{\text {th }}$ joint and $A_{p}, \omega$ remain to be constant, similar to (1).

To adaptively control the spatial frequencies between each joint i.e. the vector $\Pi=\left[\eta_{1}, \eta_{2}, \ldots, \eta_{N-1}\right]^{T}$, we propose an admittance controller shown in FIGURE 7, where $C_{p}$ is a trajectory tracking controller, $\Pi^{\boldsymbol{n}}$ are the vector of nominal spatial frequencies, $\Pi^{d}$ are the desired spatial frequencies, $\Phi^{d}$

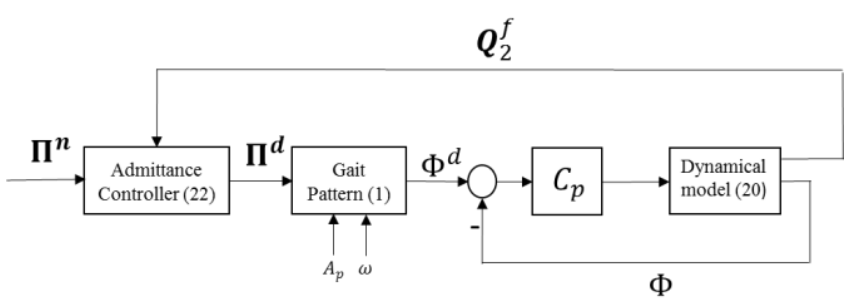

FIGURE 7: CONTROL SCHEME BLOCK DIAGRAM

is the vector of desired joint angles generated from (22) and the admittance controller is defined as follows:

$$
\begin{aligned}
M_{d}\left(\ddot{\boldsymbol{\Pi}}^{d}-\ddot{\boldsymbol{\Pi}}^{n}\right) & +D_{d}\left(\dot{\boldsymbol{\Pi}}^{d}-\dot{\boldsymbol{\Pi}}^{n}\right) \\
& +K_{d}\left(\boldsymbol{\Pi}^{d}-\boldsymbol{\Pi}^{n}\right)=\mathcal{S} \boldsymbol{Q}_{2}^{f}
\end{aligned}
$$

where $M_{d}, D_{d}$, and $K_{d}$ are positive desired inertia, damping and stiffness respectively and $S_{N-1 \times N-1}$ is gain matrix to be designed.

As can be seen in (23), choosing the nominal gait parameters $\boldsymbol{\Pi}^{\boldsymbol{n}}$, if there is no external force $\boldsymbol{Q}_{2}^{f}, \boldsymbol{\Pi}^{\boldsymbol{d}}$ converges to $\boldsymbol{\Pi}^{\boldsymbol{n}}$, however when $\boldsymbol{Q}_{2}^{f} \neq 0, \boldsymbol{\Pi}^{\boldsymbol{d}}$ deviates from its nominal value based on the feedback signal $\boldsymbol{Q}_{2}^{f}$ and dynamic (23), similar to conventional admittance controllers in [20]. However, to fully define controller (23) the gain matrix $\mathcal{S}$ should be chosen appropriately, for which we found the following structure very effective:

$$
\mathcal{S}=k_{\Pi}\left[\begin{array}{ccccc}
0 & 1 & 0 & \ldots & 0 \\
0 & 0 & 1 & \ldots & 0 \\
0 & 0 & 0 & \ldots & 0 \\
\vdots & \vdots & \vdots & \vdots & \vdots \\
0 & 0 & 0 & \ldots & 0
\end{array}\right]_{N-1 \times N-1}
$$

where $k_{\Pi}$ is a scaler value has been chosen to be 2.2 based on trial and error. It should be noted that the structure of matrix $\mathcal{S}$ is chosen in this way, so that each joint will receive the feedback signal from the joint ahead of it. Hence, except the head joint's gait parameters, the spatial frequency between other joints will be controlled based on (23). The following figure shows the robot climbing over an obstacle with the height of $10 \mathrm{~cm}$ using this strategy:

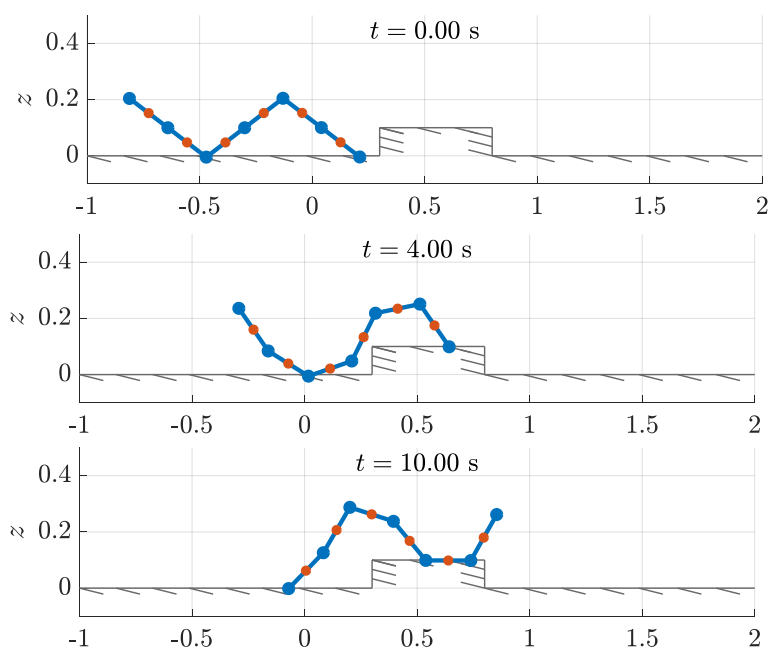



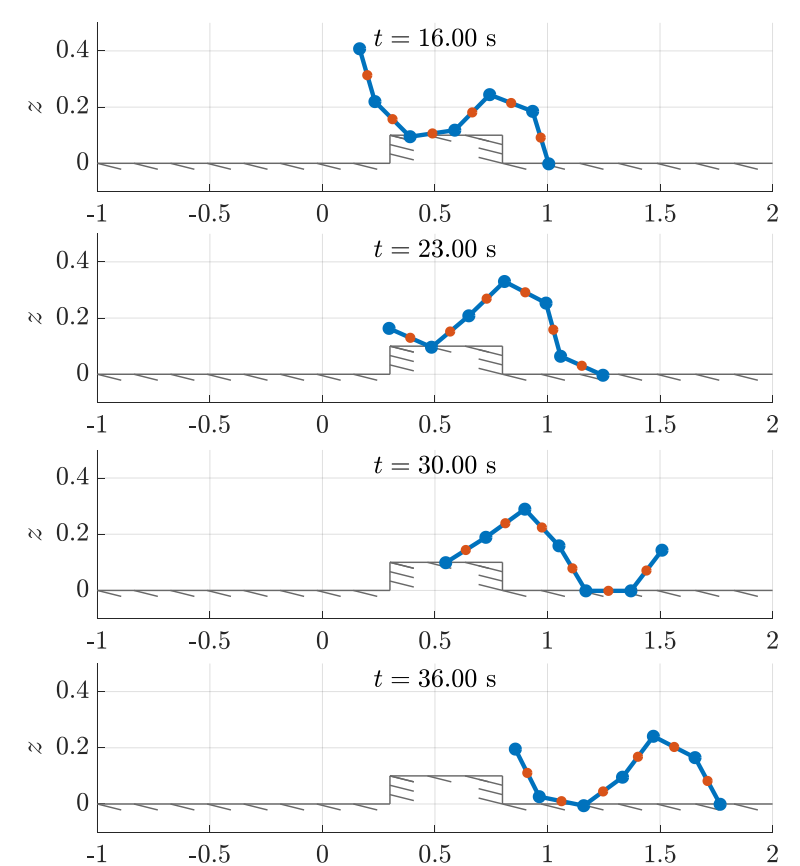

FIGURE 8: SIMULATED ADAPTIVE PEDAL WAVE MOTION

As shown in FIGURE 8, the robot successfully climbs over the obstacle located on its path without knowing the its exact location with $A_{p}=1.1 \mathrm{rad}, \omega=\frac{2 \pi}{5} \mathrm{rad} / \mathrm{sec}$ and $\Pi^{n}=$ $\left[\frac{-\pi}{2}, \frac{-\pi}{2}, \ldots, \frac{-\pi}{2}\right]$, not possible without using the admittance controller. It should be noted that to model the obstacle, the same spring damper framework is used to model the interaction between the robot and the obstacle, similar to the model used to take into account the contact between the robot and the ground.

\section{CONCLUSION}

In this paper we introduced a generalized modelling framework for modelling planar modular snake robots performing undulation in vertical and horizontal plane, also known as pedal wave and lateral undulation, respectively. We demonstrated that such a dynamical model with a spring damper contact model can easily be implemented to simulate the motion of the robot when it is subject to external forces. Moreover, we also showed that such model is powerful enough to simulate snake locomotion on uneven terrains. Additionally, an adaptive gait based controller proposed for pedal wave motion of the snake robot and simulation results shows that the robot successfully climbs over an obstacle with the height $100 \mathrm{~mm}$ without any prior knowledge about the position of the obstacle.

\section{APPENDIX}

Matrix $M$ in dynamical model (20) can be obtained as follows:

$$
\begin{aligned}
M(q)=m\left(\mathcal{B}_{1}+\mathcal{A} \mathcal{S}_{\theta} \mathcal{C}\right)^{T}\left(\mathcal{B}_{1}+\mathcal{A} \mathcal{S}_{\theta} \mathcal{C}\right) & \\
& +m\left(\mathcal{B}_{2}-\mathcal{A} \mathcal{C}_{\theta} \mathcal{C}\right)^{T}\left(\mathcal{B}_{2}-\mathcal{A C} \mathcal{C}_{\theta} \mathcal{C}\right)+j \mathcal{C}^{T} \mathcal{C}
\end{aligned}
$$

and the vector of centripetal forces in (20) can be found as below:

$$
\begin{gathered}
\boldsymbol{C}(q, \dot{q})=m\left\{B_{1}^{T} A \mathcal{C}_{\theta} \operatorname{diag}(\mathcal{C} \dot{q}) \mathcal{C}+\mathcal{C}^{T} \mathcal{C}_{\theta} \operatorname{diag}(\mathcal{C} \dot{q}) A^{T} B_{1}\right. \\
+\mathcal{C}^{T} \mathcal{S}_{\theta} A^{T} A \mathcal{C}_{\theta} \operatorname{diag}(\mathcal{C} \dot{q}) \mathcal{C} \\
\left.+\mathcal{C}^{T} \mathcal{C}_{\theta} \operatorname{diag}(\mathcal{C} \dot{q}) A^{T} A \mathcal{S}_{\theta} \mathcal{C}\right\} \dot{\boldsymbol{q}}
\end{gathered}
$$

$+m\left\{B_{2}^{T} A \mathcal{S}_{\theta} \operatorname{diag}(\mathcal{C} \dot{q}) \mathcal{C}+\mathcal{C}^{T} \mathcal{S}_{\theta} \operatorname{diag}(\mathcal{C} \dot{q}) A^{T} B_{2}-\right.$ $\left.\mathcal{C}^{T} \mathcal{C}_{\theta} A^{T} A \mathcal{S}_{\theta} \operatorname{diag}(\mathcal{C} \dot{q}) \mathcal{C}-\mathcal{C}^{T} \mathcal{S}_{\theta} \operatorname{diag}(\mathcal{C} \dot{q}) A^{T} A \mathcal{C}_{\theta} \mathcal{C}\right\} \dot{\boldsymbol{q}}$

$+\frac{m}{2}\left[\begin{array}{c}\dot{\boldsymbol{q}}^{T} B_{1}{ }^{T} A \mathcal{C}_{\theta} \operatorname{diag}\left(C_{1}\right) \mathcal{C} \dot{\boldsymbol{q}} \\ \dot{\boldsymbol{q}}^{T} B_{1}{ }^{T} A \mathcal{C}_{\theta} \operatorname{diag}\left(C_{2}\right) \mathcal{C} \dot{\boldsymbol{q}} \\ \vdots \\ \dot{\boldsymbol{q}}^{T} B_{1}{ }^{T} A \mathcal{C}_{\theta} \operatorname{diag}\left(C_{2 N+1}\right) \mathcal{C} \dot{\boldsymbol{q}}\end{array}\right]+\frac{m}{2}\left[\begin{array}{c}\dot{\boldsymbol{q}}^{T} \mathcal{C}^{T} \mathcal{C}_{\theta} \operatorname{diag}\left(\mathcal{C}_{1}\right) A^{T} B_{1} \dot{\boldsymbol{q}} \\ \dot{\boldsymbol{q}}^{T} \mathcal{C}^{T} \mathcal{C}_{\theta} \operatorname{diag}\left(\mathcal{C}_{2}\right) A^{T} B_{1} \dot{\boldsymbol{q}} \\ \vdots \\ \dot{\boldsymbol{q}}^{T} \mathcal{C}^{T} \mathcal{C}_{\theta} \operatorname{diag}\left(\mathcal{C}_{2 N+1}\right) A^{T} B_{1} \dot{\boldsymbol{q}}\end{array}\right]$ $+\frac{m}{2}\left[\begin{array}{c}\dot{\boldsymbol{q}}^{T} B_{2}{ }^{T} A \mathcal{S}_{\theta} \operatorname{diag}\left(\mathcal{C}_{1}\right) \mathcal{C} \dot{\boldsymbol{q}} \\ \dot{\boldsymbol{q}}^{\boldsymbol{T}} B_{2}{ }^{T} A \mathcal{S}_{\theta} \operatorname{diag}\left(\mathcal{C}_{2}\right) \mathcal{C} \dot{\boldsymbol{q}} \\ \vdots \\ \dot{\boldsymbol{q}}^{\boldsymbol{T}} B_{2}{ }^{T} A \mathcal{S}_{\theta} \operatorname{diag}\left(\mathcal{C}_{2 N+1}\right) \mathcal{C} \dot{\boldsymbol{q}}\end{array}\right]+\frac{m}{2}\left[\begin{array}{c}\dot{\boldsymbol{q}}^{\boldsymbol{T}} \mathcal{C}^{T} \mathcal{S}_{\theta} \operatorname{diag}\left(\mathcal{C}_{1}\right) A^{T} B_{2} \dot{\boldsymbol{q}} \\ \dot{\boldsymbol{q}}^{T} \mathcal{C}^{T} \mathcal{S}_{\theta} \operatorname{diag}\left(\mathcal{C}_{2}\right) A^{T} B_{2} \dot{\boldsymbol{q}} \\ \vdots \\ \dot{\boldsymbol{q}}^{T} \mathcal{C}^{T} \mathcal{S}_{\theta} \operatorname{diag}\left(\mathcal{C}_{2 N+1}\right) A^{T} B_{2} \dot{\boldsymbol{q}}\end{array}\right]$ $+\frac{m}{2}\left[\begin{array}{c}\dot{\boldsymbol{q}}^{T}\left(\mathcal{C}^{T} \mathcal{C}_{\theta} \operatorname{diag}\left(C_{1}\right) A^{T} M A \mathcal{S}_{\theta} \mathcal{C}+C^{T} \mathcal{S}_{\theta} A^{T} M A \mathcal{C}_{\theta} \operatorname{diag}\left(C_{1}\right) \mathcal{C}\right) \dot{\boldsymbol{q}} \\ \dot{\boldsymbol{q}}^{T}\left(\mathcal{C}^{T} \mathcal{C}_{\theta} \operatorname{diag}\left(C_{2}\right) A^{T} A \mathcal{S}_{\theta} \mathcal{C}+C^{T} \mathcal{S}_{\theta} A^{T} A \mathcal{C}_{\theta} \operatorname{diag}\left(C_{2}\right) \mathcal{C}\right) \dot{\boldsymbol{q}} \\ \vdots \\ \dot{\boldsymbol{q}}^{T}\left(\mathcal{C}^{T} \mathcal{C}_{\theta} \operatorname{diag}\left(C_{2 N+1}\right) A^{T} A \mathcal{S}_{\theta} \mathcal{C}+C^{T} \mathcal{S}_{\theta} A^{T} A \mathcal{C}_{\theta} \operatorname{diag}\left(C_{2 N+1}\right) \mathcal{C}\right) \dot{\boldsymbol{q}}\end{array}\right]$ $-\frac{m}{2}\left[\begin{array}{c}\dot{\boldsymbol{q}}^{T}\left(\mathcal{C}^{T} \mathcal{S}_{\theta} \operatorname{diag}\left(C_{1}\right) A^{T} A \mathcal{C}_{\theta} \mathcal{C}+\mathcal{C}^{T} \mathcal{C}_{\theta} A^{T} A \mathcal{S}_{\theta} \operatorname{diag}\left(C_{1}\right) \mathcal{C}\right) \dot{\boldsymbol{q}} \\ \dot{\boldsymbol{q}}^{T}\left(\mathcal{C}^{T} \mathcal{S}_{\theta} \operatorname{diag}\left(C_{2}\right) A^{T} A \mathcal{C}_{\theta} \mathcal{C}+\mathcal{C}^{T} \mathcal{C}_{\theta} A^{T} A \mathcal{S}_{\theta} \operatorname{diag}\left(C_{2}\right) \mathcal{C}\right) \dot{\boldsymbol{q}} \\ \vdots \\ \dot{\boldsymbol{q}}^{T}\left(\mathcal{C}^{T} \mathcal{S}_{\theta} \operatorname{diag}\left(C_{2 N+1}\right) A^{T} A \mathcal{C}_{\theta} \mathcal{C}+\mathcal{C}^{T} \mathcal{C}_{\theta} A^{T} A \mathcal{S}_{\theta} \operatorname{diag}\left(C_{2 N+1}\right) \mathcal{C}\right) \dot{\boldsymbol{q}}\end{array}\right]$

where

$$
\boldsymbol{C}_{N \times(N+2)}=\left[\begin{array}{lll}
\mathcal{F} & \boldsymbol{V} & \mathbf{0}_{N \times 2}
\end{array}\right]=\left[\boldsymbol{C}_{1}, \boldsymbol{C}_{2}, \ldots, \boldsymbol{C}_{N+2}\right]
$$

\section{REFERENCES}

[1] Hirose, S., 1993, "Biologically Inspired Robot," Oxford Univ. Press.

[2] Rollinson, D., and Choset, H., 2016, "Pipe Network Locomotion with a Snake Robot," J. F. Robot., 33(3), pp. 322-336.

[3] Yamada, H., Takaoka, S., and Hirose, S., 2013, "A Snake-like Robot for Real-World Inspection Applications (the Design and Control of a Practical Active Cord Mechanism)," Adv. Robot., 27(1), pp. 4760.

[4] Liljebäck, P., Pettersen, K. Y., Stavdahl, O., and Gravdahl, J. T., 2011, "Experimental Investigation of Obstacle-Aided Locomotion with a Snake Robot," IEEE Trans. Robot., 27(4)

Hu, D. L., Nirody, J., Scott, T., and Shelley, M. J., 2009, "The Mechanics of Slithering Locomotion," Proc. Natl. Acad. Sci. U. S. A., 106(25).

[6] Marvi, H., Bridges, J., Hu, D. L,, 2013, “Snakes Mimic Earthworms: Propulsion Using Rectilinear Travelling Waves.,” J. R. Soc. Interface, 10(84), p. 20130188.

Yamada, H., and Hirose, S., 2010, "Steering of Pedal Wave of a Snake-like Robot by Superposition of Curvatures," 2010 IEEE/RSJ International Conference on Intelligent Robots and Systems, IEEE, pp. 419-424.

Tanaka, M., and Matsuno, F., 2014, "Control of Snake Robots with Switching Constraints: Trajectory Tracking with Moving Obstacle," Adv. Robot., 28(6), pp. 415-429.

[9] Pettersen, K. Y., 2017, “Snake Robots,” Annu. Rev. Control, 44, pp. 19-44. 
[10] Filippov, A. E., and Gorb, S. N., 2016, "Modelling of the Frictional Behaviour of the Snake Skin Covered by Anisotropic Surface Nanostructures," Sci. Rep., 6(August 2015), p. 23539.

[11] Liljebäck, P., Pettersen, K. Y., Stavdahl, O., and Gravdahl, J. T., 2010, "Hybrid Modelling and Control of Obstacle-Aided Snake Robot Locomotion," IEEE Trans. Robot., 26(5).

[12] Transeth, A. A., Leine, R. I., Glocker, C., Pettersen, K. Y., and Liljebäck, P., 2008, "Snake Robot ObstacleAided Locomotion: Modeling, Simulations, and Experiments," IEEE Trans. Robot., 24(1).

[13] Li, G., Li, W., Zhang, J., and Zhang, H., 2015, "Analysis and Design of Asymmetric Oscillation for Caterpillar-Like Locomotion,” J. Bionic Eng., 12(2), pp. 190-203.

[14] Chen, L., Ma, S., Wang, Y., Li, B., and Duan, D., 2007, "Design and Modelling of a Snake Robot in Traveling Wave Locomotion," Mech. Mach. Theory, 42(12), pp. 1632-1642.

[15] Akbarzadeh, A., and Kalani, H., 2012, "Design and Modeling of a Snake Robot Based on Worm-Like Locomotion," Adv. Robot., 26(5-6), pp. 537-560.

[16] Kano, T., Watanabe, Y., Satake, F., and Ishiguro, A., 2014, "Decentralized-Controlled Multi-Terrain Robot Inspired by Flatworm Locomotion," Adv. Robot., 28(7), pp. 523-531.

[17] Bogert, C. M., 1947, "Rectilinear Locomotion in Snakes," Copeia, 1947(4), pp. 253-254.

[18] Javaheri Koopaee, M., Bal, S., Pretty, C., and Chen, X., 2018, "Design and Development of a Cost-Effective Snake Robot with Active Stiffness Control for Locomotion on Uneven Terrain," Manuscr. Submitt. Publ.

[19] Whitman, J., Ruscelli, F., Travers, M., and Choset, H., 2016, "Shape-Based Compliant Control with Variable Coordination Centralization on a Snake Robot," (Cdc), pp. 5165-5170.

[20] Ott, C., Mukherjee, R., and Nakamura, Y., 2010, "Unified Impedance and Admittance Control," Robot. Autom. (ICRA), 2010 IEEE Int. Conf., pp. 554-561. 